\title{
Cross-border and digital socialization of personality
}

\author{
Angelica Luchinkina ${ }^{1,{ }^{*}}$, Lilia Zhikhareva $^{1}$, Tatiana Yudeeva $^{1}$ and Irina Luchinkina ${ }^{1}$ \\ ${ }^{1}$ Crimean Engineering and Pedagogical University, Simferopol, 295015, Russia
}

\begin{abstract}
The article discusses the problem of the influence of digital space and the digital environment on the cognitive processes of primary school children and adolescents. The authors cite and theoretically analyze the characteristics of a personality included in digital space. The contradictions in the ideas about the influence of digital space on the personality are noted: on the one hand, digital space can have a negative impact on the formation of cognitive processes, on the other hand, stimulate their development. The authors empirically investigated such parameters as verbal and logical thinking, behavioral features in the digital environment, attention characteristics and strategies for overcoming stressful situations. It was revealed that the respondents of all groups of the sample have developed classification skills rather than the skills of establishing semantic analogies. The results of the study showed that adolescents with high Internet activity choose impulsive, manipulative and aggressive actions as coping strategies, less often they choose such as seeking social support and cautious steps.
\end{abstract}

\section{Introduction}

\subsection{Relevance}

The world of postmodernity is rapidly developing, raising a tsunami of information that is not assimilated by the human brain, destroys the barriers of memory and perception. Society is rapidly moving away from technogenic to technocracy, acquiring new opportunities and new problems along this way. In the desire to cover all information flows, a person striving for multitasking delves into information and digital diversity.

The result is distorted cognitive processes leading to passive perception of information or difficulties in processing this information. Clip thinking has become the main metaphor of the last decade. Against the background of mass computerization, the concepts of education are changing. If 20 years ago humanity was distrustful of the very possibility of distance education, then today distance education is as real as the traditional form of education.

\footnotetext{
* Corresponding author: aluch@yandex.ru
} 


\subsection{Statement of the primary material}

There are several approaches to the question of gender differences in the aggressive behavior of adolescents: psychophysiological, psychosocial, and socio-psychological. (нет в оригинале)

Dr. Arik Sigman (2007), an international expert on time management spent by children and adolescents in front of screen devices, noted that young children, who have up to $80 \%$ of their brains formed in the first three years of life, should be in contact with the real world and physical objects. Among the consequences of excessive use of gadgets by children and adolescents, Dr. Arik Sigman named obesity and the development of cancer, depression, anxiety, decreased interpersonal communication skills and poor academic performance.

The research carried out by O. Grigoriev, Doctor of biological Sciences, Professor, Chairman of the Russian National Committee for Protection against Non-Ionizing Radiation, looks frightening. Therefore, the number of sick children has significantly increased over the past decade and a half, which means that the number of healthy children has decreased (Grigoriev, 2013). In addition, according to scientists, screen addiction leads to the child's inability to concentrate on any activity, hyperactivity, increased distraction, delayed speech development, increased aggressiveness and cruelty.

Another problem is related to the virtualization of children, adolescents and youth. Modern information and communication technologies allow to make the living space as convenient as possible: young people do not need to go to the library to read a book, they can meet friends on Skype, Discord and other social networks. Food and clothing can be ordered. You can also take a walk on the Internet. Any game can be stopped and returned to its original positions (Luchinkina, 2012).

Eternal values are depreciated: good and evil, life and death. You can delete a person from friends on social networks and then return them. Young people have changed their attitude to time and space. The principle of everything at once, here and now, becomes fundamental in communication. Young people get much more degrees of freedom than 1520 years ago. At the same time, the changed system of values reflects the very concept of self-responsibility. The number of young people whose freedom and responsibility are in conflict with each other is increasing. Being responsible means not being free (Luchinkina, 2019).

What did it lead to? First, to sense of loneliness and alienation among adolescents and young people aged 13-18 years. There are many more children in classes and groups outside the social circle than 10 years ago. By definition, adolescents and young people cannot exist outside of the collective, outside of the reference group. Maybe that's why there are more frequent cases of young people joining groups with an antisocial orientation. Moreover, it is precisely the desire of young people and adolescents to be in demand and necessary that is used not only in political actions by individual politicians, but also for extremist and terrorist purposes (Luchinkina, 2019).

Another resulting problem is the increasing weight of individual achievement values in relation to collective values. Personal values do not form feelings of Motherland, friendship, love.

The passion for gadgets, the desire for more vivid images is expressed in the digital environment, which is a combination of technical achievements, virtual platforms and the socio-cultural reality based on them. The digital environment, including the Internet and other technical realities, itself becomes an environment of a person socialization. The ambiguity of the influence of digital reality on a person is manifested in the fact that new possibilities of digitalization (the speed and brightness of information, its portioned supply and, in this regard, the transformation of the main types of activities (communication, 
education, game, creativity, recreation, professional, educational and scientific activities) can lead to both personal growth and personal deviations (Luchinkina, 2018).

The problem is that attempts to transfer the socialization model to the digital environment do not make it possible to understand the psychological laws of digital socialization of the individual as a whole.

Various aspects of the processes unfolding in the digital environment have been actively studied in recent decades: Internet socialization (Luchinkina, 2012); cyber socialization (Pleshakov, 2013); virtual socialization (Skuratov, 2016); informational socialization (Castells 2016; Rakitov 2013); media socialization (Petrunko, 2010). These studies do not fully describe the processes taking place in the digital environment, since scientists associate their research with the Internet, the emergence of which is due to a person's desire to create alternative worlds (Suler, 2015). The processes taking place in the digital environment are much broader, because they include not only the possibility of creating alternative worlds, but also the process of human interaction with technology, the digitalization of activities, and the transformation of this activity into a digital space.

Sociological research (Bell 2007; Castells 2016) examines the communicative aspects of the digital environment, including the possibility of creating a unified communication field. Philosophical research (Prokudin, 2013) concerns the consideration of the transformation of values, behavioral and communicative models of personality, language and thinking under the influence of digitalization.

Studies of the digital environment reveal some personality characteristics that relate to the behavioral and communicative sphere (Galkin 2019; Sergeeva 2016); the cognitive sphere; and the value sphere (Prokudin, 2013).

Recognizing the importance of these studies, focus on the fact that they examine the separate influence of digital environment on personality; however, this does not allow us to see a complete picture of digital socialization of the individual and to reveal the concept of digital personality in general.

In turn, Stefaneas, P. (2013) and Vandoulakis I. M. (2013) concluded that the digital space contributed to the formation of cognitive neoplasms. The usual system of proof is mathematical. As a rule, in mathematical practice, a proof indicates the logic of a statement based on previously defined logics of other statements.

Being actively in the digital environment affects brain activity, higher mental processes and, as a result, the personality as a whole (Lombina, 2018). For example, a child unaccustomed to the perception of visual information has difficulty in understanding text and oral speech. The researcher notes that, first of all, the processes of perception suffer, and in the future, problems are noted with thinking.

Taylor M., in contrast to Lombina T.N., note that modern children are already active digital users and the digital environment can influence them in different ways: on the one hand, to form passive thinking and perception, on the other - to develop and stimulate cognitive neoplasms (Taylor, 2006). Moreover, the classics of pedagogy have repeatedly noted, that any socializing space is ambivalent.

T. Compernolle writes that a preschool age child, finding himself in digital diversity, becomes dependent on sound, color, picture movements and games. According to the author's point of view, this dependence is reinforced by a small release of dopamine that occurs when a virtual stimulus is presented (Compernolle, 2014). These conclusions are important in the context of the study of psychophysiological changes under the influence of the digital environment; however, it is necessary to clarify a number of nuances. Firstly, the preschooler's increased need for sound, color and picture movements is fully justified by their age characteristics. It is with the help of these reinforcers that attention and cognitive activity are formed. 
Second, virtual children's games, as a rule, are designed for a certain age and level of development (in Google Play and the App Store: "Colors and Shapes", "Children's Puzzles", "Coloring Books for Children", "Lesson: Music and Drawing" etc.). Most often, these games are both entertaining and educating. Third, this study refers more to the virtual space than to the digital one.

Digital personality, according to scientists, is the result of digitizing personal data, needs, activities and relationships (Soldatova 2019, Voiskunsky 2019). Attempts to study the characteristics of virtual personality in connection with changes in the functioning of the cognitive sphere are noted in the works of R.V. Ershova (Ershova, 2019). A number of researchers have noted that a digital personality is one who is prone to Internet addiction. However, we assume that within the framework of the activity of the digital personality, one cannot talk about addiction, but rather about the transfer of this activity to the digital space, which is convenient and appropriate according to the progress of modern society. Studies of affective and motivational characteristics of the virtual personality were not taken into account by the researchers. Note that cognitive, behavioral and psychophysiological features have not been sufficiently studied. A holistic picture of the virtual personality, as well as its possible typology, was not offered.

Thus, the subject of research is the cognitive processes of the personality (perception, memory, and thinking), social behavior strategies.

The main goal of this research is to study the features of cognitive processes of the Internet-active personality and its behavior.

\section{Materials and Methods}

Due to the age peculiarities, different methods were used for primary schoolchildren and adolescents. However, the goals were similar. At the same time, the fact that the activity of younger schoolchildren in the virtual space is more often associated with games was taken into account. Note that Internet activity is a type of digital behavior.

In the empirical study, a set of psychodiagnostic techniques, that corresponded to the objectives of the study and the age of the subjects, was used. To study the peculiarities of thinking of primary schoolchildren, the following methods were used: "Definition of concepts, clarification of reasons, identification of similarities and differences in objects"; "Exclusion of words"; "Revealing general concepts"; "Excluding unnecessary"; Lippmann test "Logical patterns". To diagnose attention, correction tests were used. To study the characteristics of behavior in the digital environment - content analysis of pages in social networks.

As a psychodiagnostic toolkit for adolescents, the following methods were used: to determine the current level of mental development and intelligence - the School test of mental development-2, Weksler's test; to determine the peculiarities of attention - the proofreading test of $\mathrm{B}$. Bourdon; to determine behavior strategies - the methodology «Strategic Approach to Coping Scale (SACS)" by S. Hobfall (adapted by N.E. Vodopyanova, E.S. Starchenkova).

The main objectives of the presented empirical study were the distribution of subjects into groups with different online activity; determination of the characteristics of cognitive functions in children and adolescents with different digital activity; determination of social behavior strategies in adolescents with different digital and online activity.

Thus, the subjects were divided into two groups depending on the time of using the gadgets. The time of digital activity among adolescents was determined not only by being on social networks, searching for information, publishing and reading posts, but also by communicating in instant messengers (WhatsApp, Viber), viewing and posting photos, Instagram posts, online games, etc. 
The study involved 120 teenagers aged 13-15 years and 84 primary school children. The study was conducted based on secondary schools in Simferopol, Republic of Crimea.

\section{Results}

The results of the distribution of children and adolescents into groups with different digital activity made it possible to distinguish two groups of subjects: group 1 (55 adolescents and 56 younger schoolchildren) consisted of children and adolescents with online activity and using gadgets up to 4 hours a day. In turn, group 2 (65 adolescents and 28 younger schoolchildren) consisted of children and adolescents with digital activity 6-8 hours a day or more.

Thus, it was found that surfing the Internet in general does not have a negative effect on the development of cognitive abilities of schoolchildren (Figure 1).

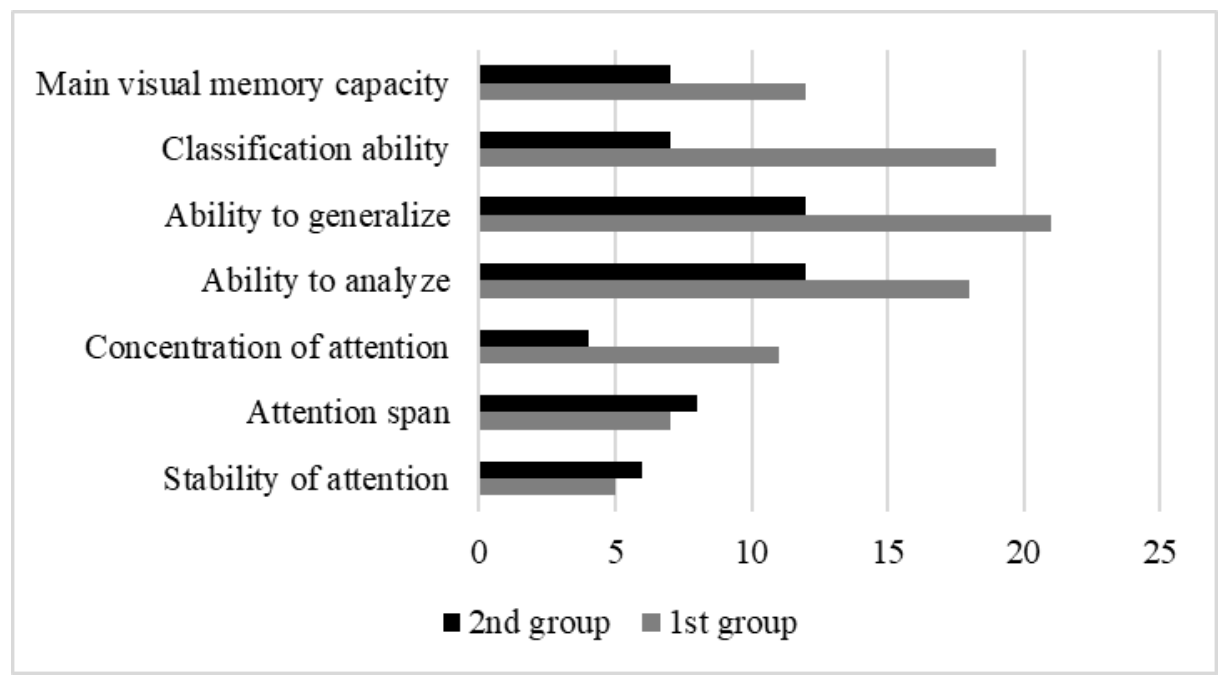

Fig. 1. Indicators of research on the characteristics of the cognitive sphere of primary schoolchildren.

The results of the study make it possible to assume that frequent access to gaming activity in the Internet, to a greater extent, requires a certain speed of mental operations and adherence to the strategy of the game, but to a lesser extent, such a side of thinking as the ability to classify is involved.

Analysis of the research results showed (according to the methodology of the School test of mental development) that the ability to classify, both in group 1 and in group 2 of subjects, is developed to a greater extent (38\% and $50 \%$, respectively) than the ability to build analogies and generalize (Figure 2). It should be noted that the average percentage of subtests being completed is less than $50 \%$, which may indicate a low level of development of mental operations in adolescents. 


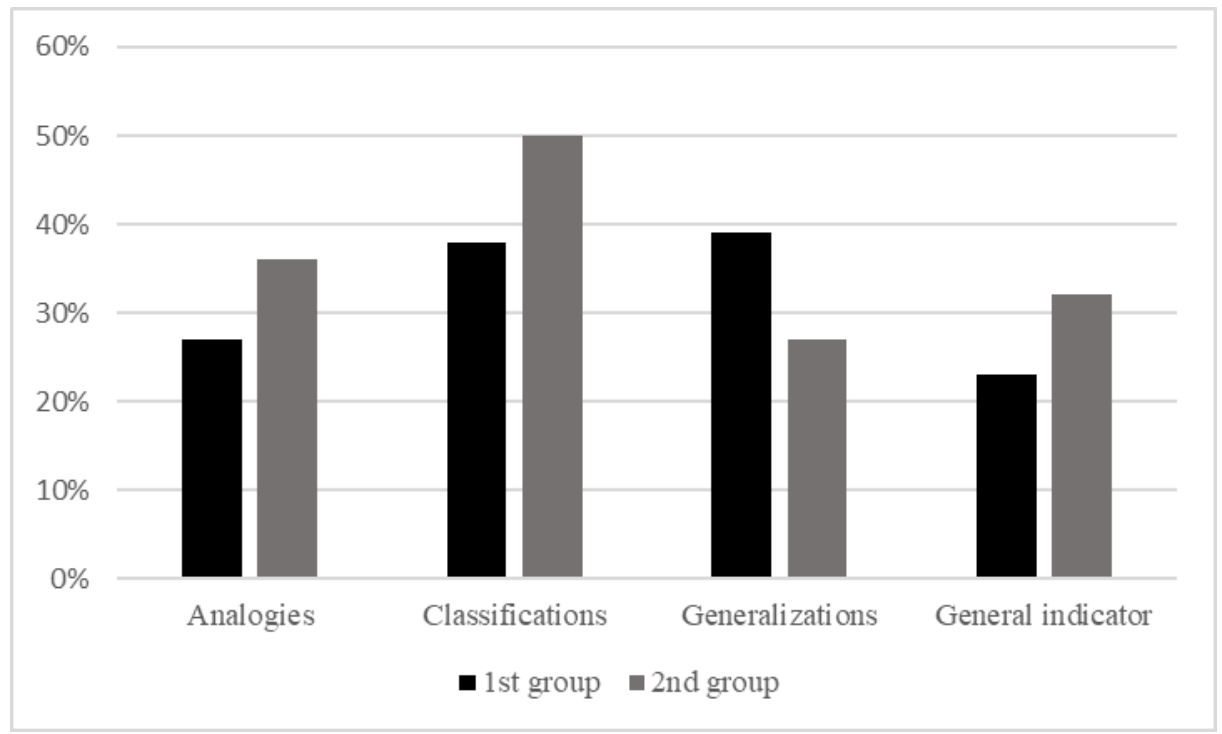

Fig. 2. Indicators of the development of cognitive functions in adolescents.

The results of statistical analysis indicate the absence of significant differences, which may be due to the fact that the cognitive sphere in adolescence is already sufficiently developed and less associated with the digital activity of an adolescent. It can also be assumed that in the new conditions of digitalization and the rapid spread of digital technologies, normative changes in personal and cognitive development in modern adolescents are possible.

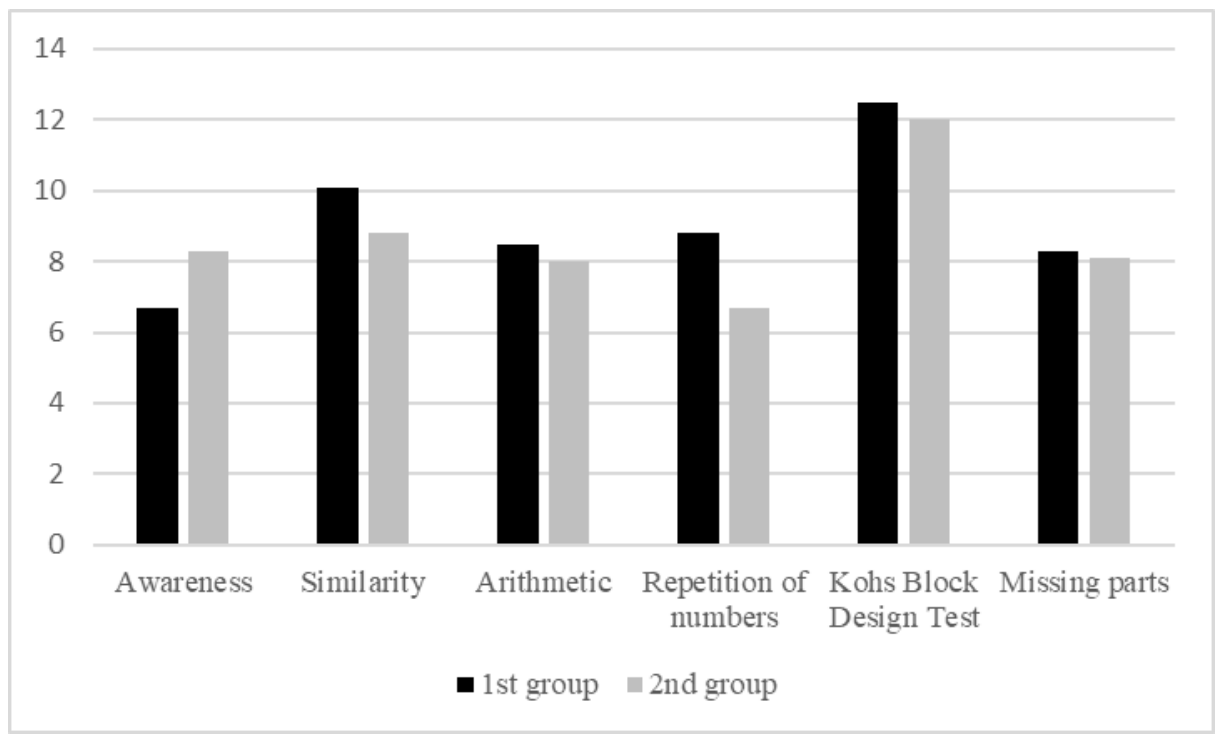

Fig. 3. Indicators of the development of cognitive functions of adolescents (according to the Wechsler test).

Statistical analysis of the results obtained (the Wechsler test) did not reveal significantly significant differences between the groups according to the Awareness subtest. This may indicate that adolescents of both groups have approximately the same amount of perceptual attention and the level of perceptual abilities aimed at differentiating parts in visual images. 
Adolescents with high digital activity have difficulties in classification, in comparison, in generalization and ordering information, they have a lower level of development of conceptual thinking. Also, statistical analysis of the obtained results did not reveal significantly significant differences in the Arithmetic and Kohs Block subtests, however, the indicators online active adolescents are lower, thus, the subjects of group 1 are more able to cope with tasks on spatial imagination and thinking. The results obtained for the "Repetition of Numbers" subtest indicate that adolescents with low online activity up to 4 hours a day have better developed RAM and active attention than adolescents with high digital activity.

As behavioral strategies, primary school students in the first group use "entering into social contact" $(p<0.05)$ and "seeking social support" $(p<0.05)$, while the subjects in the second group use impulsive and aggressive strategies $(p<0,05)$.

Evaluation of social behavior strategies in adolescents with different digital activity showed that adolescents in the group 1 also more often use the prosocial coping strategies "entering into social contact" $(p<0.05)$ and "seeking social support" $(p<0.05)$. Adolescent with high digital activity are more likely to use impulsive $(\mathrm{p}<0.05)$, manipulative $(\mathrm{p}<0.05)$ and aggressive actions $(\mathrm{p}<0.05)$ as coping strategies. However, among them there were adolescents who also use strategies such as "seeking social support" and "taking caution."

\section{Discussion}

The results of the pilot study make it possible to put forward the assumption that the level of digital online activity and digital technologies can contribute to a certain extent to the more successful development of cognitive functions.

However, the obtained results and patterns require further in-depth analysis. Therefore, the question remains unanswered, what is the reason that the most Internet-active children develop their mental operations better in primary school age, while in adolescence their development slows down with increasing Internet activity. Earlier studies by other authors have noted the negative impact of the digital environment on the development of cognitive and social strategies of the individual. However, as the results of our study have shown, in primary school age, cognitive strategies of digital children are formed at a higher level than of those who spend less time in the digital environment.

It calls for further research and the choice of the second group of social strategies by the respondents. The obtained results allow us to formulate an assumption that the higher the level of digital and online activity (uncontrolled activity), the more often adolescents will use non-constructive coping strategies, have a low level of self-control and regulation of their actions.

\section{Conclusions}

The results of the study led to the following conclusions:

1. Digital socialization of children and adolescents is a cross-border phenomenon of the modern era and its result in the formation of a digital personality. Digital identity is the result of digitizing personal data, needs, activities and relationships. A virtual personality is a manifestation of a digital personality in a virtual space. The uncertainty of the categorical apparatus and the incompleteness of definitions require further research regarding the process of socialization of a person in the digital space and socialization in general.

2. Socio-cultural practices of the digital environment are striking in their diversity and continue to develop thanks to "digital" users (a continuous process-culture is superimposed on culture, replacing some existing ideals with others). Moreover, if changes in the 
functioning of mental processes in some people are suggested by a number of scientists, then the question of the invariability of those same processes in others remains open.

3. The development of cognitive processes of the digital personality in age dynamics depending on the degree of digital (Internet) activity is a poorly studied process and requires additional research.

\section{References}

1. A. Sigman, Remotely Controlled: How television is damaging our lives (Vermilion, London, 2007)

2. Yu.G. Grigoriev, O.A. Grigoriev, Cellular and health. Electromagnetic environment, radiobiological and hygienic problems (Economics, Moscow, 2013)

3. A.I. Luchinkina, Human psychology on the Internet. Information Systems (Kiev, 2012)

4. A.I. Luchinkina, I.S. Luchinkina, Russian psychological journal 1(16), 128-143 (2019) doi: 10.21702/rpj.2019.1.6

5. I.S. Luchinkina, Humanitarian sciences 4(44), 153-158 (2018)

6. V.A. Pleshakov, Bulletin of KGU named after N.A. Nekrasov 19, 117-120 (2013)

7. A.B. Skuratov, Social sciences and humanities 16, 79-82 (2016)

8. M. Castells, Power of communication (GU VSHE, Moscow, 2016)

9. A.I. Rakitov, Philosophy of the computer revolution (Direct-Media, Moscow, 2013)

10. O.V. Petrunko, Children and media: socialization in an aggressive media environment: a monograph (Ukrpromtorservice, Poltava, 2010)

11. R.J. Suler, Psychology of the Digital Age: Humans Become Electric (Cambridge University Press, Cambridge, 2015)

12. D. Bell, V. Inozemtsev, The era of disunity. Center for Research on Post-Industrial Society (Moscow, 2007)

13. D.E. Prokudin, Philosophy of Russian statehood: history and modernity 1, 324-332 (2013)

14. D.V. Galkin, E.Yu. Baboshko, Culturology and art history 33, 5-14 (2019)

15. I.L. Sergeeva, Culture and civilization 6, 55-65 (2016)

16. P. Stefaneas, I.M Vandoulakis, Philosophical Engineering: Toward a Philosophy of the Web (Metaphilosophy) (Wiley-Blackwell, New Jersey, 2013)

17. T.N. Lombina, O.V. Yurchenko, Society: sociology, psychology, pedagogy 1, 45-50 (2018)

18. M.L. Taylor, A Collection of Papers on Self-Study and Institutional Improvement 2(2), 48-55 (2006)

19. T. Compernolle, BrainChains: Discover your brain, to unleash its full potential in a hyperconnected, multitasking world (Compublications, Belgium, 2014)

20. G.U. Soldatova, Social psychology and society 9(3), 71-80 (2018)

21. A.E. Voiskunsky, G.U. Soldatova, Counseling psychology and psychotherapy 27(3), 22-43 (2019)

22. R.V. Ershova, Philosophical Sciences 62(2), 96-108 (2019) 